\title{
PROPUESTAS PARA LA INTERPRETACIÓN DE BOTORRITA I
}

The author thinks that the great Celtiberic inscription of Botorrita deals with a lex sacra concerning the holy domain (percuneta) of Tiricanta and he gives several proposals in order to interpret some difficult passages of this inscription. He points out some prohibitions and restrictions about the use of the domain. The author thinks that arestalo refers to the inscription itself. Other proposals are also important for the study of the tenses and moods of the Celtiberic language.

\section{Propósito de estas páginas}

La lectura, entre otra bibliografía, de la nueva interpretación de Wolfgang Meid de la primera inscripción de Botorrita ${ }^{1}$ ha renovado mi interés por este excepcional documento, al que ya dediqué un intento de interpretación hace bastantes años ${ }^{2}$.

Esta nueva bibliografia va perfeccionando cada vez más la lectura de la inscripción y va acercándonos a la interpretación de la misma como una lex sacra más o menos semejante a la del cippus Abellanus y la inscripción griega del templo de Atena Alea en Tegea ${ }^{3}$. Se trata de regulaciones de un témenos o recinto sagrado en torno a un templo de dos divinidades, común a varias localidades: regulaciones que consisten ya en prohibiciones con las multas consiguientes, ya en determinadas exigencias, ya en permisos de utilización en ciertas condiciones.

Nada objeto, pues, a las líneas generales de la interpretación de Meid, que une aportaciones propias a otras anteriores, aunque en mi reseña del libro,

1 Wolfgang Meid, Die erste Botorrita-Inschrift. Interpretation eines keltiberischen Sprachdenkmals. Innsbruck 1993. Véase mi reseña en este mismo número de EMERITA.

2 "Aportaciones a la interpretación del bronce de Botorrita", en Actas del I Coloquio sobre lenguas y culturas prerromanas de la Peninsula Ibérica, Salamanca 1976, pp. 25-47.

${ }^{3}$ Entre otras. Esta interpretación está ya en J. de Hoz-L. Michelena, La inscripción celtibérica de Botorrita, Salamanca 1974, p. 96 s. Aquí y en J. F. Eska, "The verbal desinence -Tus in the Hispano-Celtic Inscription of Botorrita", ZCPh 43, 1989, pp. 214-222, se ofrecen paralelos epigráficos latinos. 
citada en nota 1, presenté algunas pequeñas críticas y dudas. Pero pienso que, en ocasiones, otros autores presentan mejores propuestas y, en otras todavía, yo mismo puedo presentar algunas que considero preferibles.

Las páginas que siguen no estudian, pues, la inscripción paso a paso para ofrecer una nueva interpretación. Ni lo que es doctrina común ni aquello a lo que nada puedo aportar recibe atención en este artículo. Toca solamente algunos puntos en que pienso que la interpretación de Meid, personal o heredada de otros según los casos, puede ser mejorada. Me ocupo, pues, solamente de algunas palabras de algunas de las «unidades de sentido» en que Meid ha dividido el texto de la inscripción. Y distingo entre propuestas que considero verosímiles y otras que son meras posibilidades.

\section{Percunetacam y la unidad I de la inscripción}

Doy el texto de Meid de la inscripción seguido de la interpretación de este autor, la anterior a ella de F. Villar ${ }^{4}$ y la mía propia, que anticipo:

Celt.: tiricantam percunetacam tocoitoścue śarniciocue śua compalces nelitom.

Meid: «betreffend das 'bergige' Gebiet des Togoit- und des Sarnicios wurde folgendes verfügt als nicht erlaubt».

Villar: «en relación con el trescantos (llamado) Berkunetaca de Tokoits y Sarnicio así (es) el acuerdo». NOTA. Villar deja en duda si los dos genitivos dependen de «Berkunetaca» o de «acuerdo». Y deja el nelitom final fuera de su estudio.

Adrados: Tricanta (= Trifinium uel Triuium) lucum Togoitisque Sarniciique. Ita senatus. Nefas:

Creo que la interpretación de Villar, seguida en parte por Meid, es superior a la de éste. La sigue Meid al aceptar que los acusativos iniciales no son adverbiales, puesto que no hay verbo: son acusativos de relación, dice, que indican, simplemente, el tema. No está descaminada la idea, pero yo preferiría hablar de un Ac. adverbializado o de espacio o, simplemente, neutralizado en la oposición al N., como el Ac. que aparece en latín (igual que el N.) en enumeraciones e intitulaciones ${ }^{5}$. En el caso de los topónimos, este Ac. es el que habitualmente produjo sus formas románicas. Por su parte, el N. de un topónimo que, en cabeza de una inscripción, indica el tema de la misma, es especialmente frecuente en micénico (tipo Pu-ro 'Pilos').

\footnotetext{
${ }^{4}$ F. Villar, «La línea inicial del bronce de Botorrita», en Studia indogermanica et palaeohispanica in honorem A. Tovar et L. Michelena, Salamanca 1990, pp. 375-392.

${ }^{5}$ Ejemplos en M. Bassols de Climent, Sintaxis Latina I, Madrid 1963, p. 61 ss.: multa uasa... patenas aureas (en una enumeración), oliuas colonbares (en la pared de una vasija).
} 
Pero es extraño que Meid, después de aceptar de Villar que Tiricantam es 'Tres caminos' o 'Tres cantos' piense que se ha banalizado hasta significar 'área': en ejemplos que citan ambos autores es eso, un topónimo. Y Villar argumenta muy bien en ese sentido. Por supuesto, percunetacam o es un adjetivo en ${ }^{*} k o-/{ }^{*} k \bar{a}$ - concertado con la palabra anterior o es un nombre apuesto a la misma. Y los Gen. que siguen, referidos a dos dioses como hoy se reconoce generalmente, dependen bien del sintagma Tiricantam percunetacam (nombre + adj.) bien de percunetacam simplemente (si es un nombre apuesto).

Efectivamente, una vez establecido que compalces, sea verdadera la que sea de entre las etimologías propuestas ${ }^{6}$, es el 'senado' o algún cuerpo semejante (lo que es obvio tras la interpretación de 11 compalcores como un compuesto con * rēgs, véase al final de este trabajo), es bien claro que los Gen. de los dos teónimos no pueden depender de esta palabra, sino de la totalidad o de una parte del sintagma inicial en Ac.

Esto nos lleva, definitivamente, a abandonar el intento de entender la primera línea del bronce como un complejo oracional: imposible, no hay verbo. Lo que hay es una indicación local (el tema o «tópico» seguida de dos frases nominales con «es» elíptico.

La segunda es nelitom 'no es lícito', como tras Schmidt acepta Meid: evidentemente, es una prohibición general, especificada luego en tres frases con necue...litom. La primera, que traduzco por ita senatus, no ofrece problema. Pero, ¿qué opinar de la indicación o «tema» inicial, referente al lugar cuyo uso regula toda la inscripción?

Para Villar, percunetacam es un «adjetivo derivado de un topónimo derivado de un compuesto". Pero su traducción, 'el trescantos (llamado) Berkunetaka', lo hace más bien un nombre en aposición. Es lo que pienso. Pero si Tiricantam es un topónimo, como Villar demuestra bien, entonces percunetacam, sustantivación de un adjetivo en ${ }^{*}-k o /{ }^{*} k \bar{a}$-, es el nombre genérico que define $o$ clasifica al topónimo. Para mí, viene a equivaler a lat. lucum o al celta nemeto, un bosque sagrado de los dos dioses que a continuación se indican?

' La más verosímil es la que arranca de celta *balco- 'fuerte', bien testimoniada, cf. Adrados, p. 34 y Meid, p. 92. Se trataría de los hombres de autoridad (gr. oi ßé quizá los magistrados relacionados en la cara B y calificados de bintis 'magistrado' (cf. A. Beltrán-A. Tovar, Contrebia Balaisca, Zaragoza 1982, p. 77). Para mí, esta raiz aparece

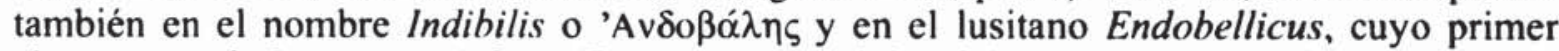
elemento es indoeuropeo (cf. A. Tovar, "A propósito del vascuence "mando" y "beltz" y los nombres de Indibil y Mandonio", en Homenaje a D. Julio Urquijo, San Sebastián 1949, p. 109 ss., que para el segundo propone un ibero-africano beltz 'negro'); e incluso, quizá, en el nombre de Contrebia Belaisca.

7 En realidad, el sentido ha pasado a ser el de témenos o 'recinto sagrado' en general: incluye campos de cereal, lo que se deduce tanto de la inscripción como de los datos arqueológicos (cf. A. Beltrán-A. Tovar, cit., pp. 18, 35, etc.). Todo este recinto estaba 
No creo, pues, en la traducción de Meid de las dos palabras iniciales como 'bergige Gebiet'. Y si, devolviendo la primera palabra a su condición de topónimo, decimos, 'la montañosa T. de Togoits y Sarnicios', esto tampoco encaja. Percunetacam, insisto, tiene que ser un nombre genérico: un derivado en ${ }^{*}-k \bar{a}$, sustantivado, de percuneta. Es 'algo, un lugar de la (o relativo a la) percuneta'.

$\mathrm{Y}$ ¿qué es percuneta? La derivación a partir de *bhergh 'elevado' (aquí coinciden Villar y Meid) debe rechazarse. No sólo el comienzo de la inscripción resulta algo completamente banal, un 'área montañosa', o algo que se renuncia a entender ('la B. de T. y S.'). Además, ocurre que con esa raíz no hay formas en $-u$ (la que aduce Villar, het. parku 'alto, elevado', él mismo reconoce que tiene un vocalismo anómalo). Meid bien lo reconoce a su vez cuando (p. 103) ha de aducir gót. fairguni, celt. Hercynia (silua), para añadir que no tienen nada que ver porque vienen de * perk $^{\mu}$. Extraña argumentación.

Percuneta, propongo, viene precisamente de *perku o perk" ${ }^{4} \bar{a}$, 'roble', 'encina' (aunque la raiz ha pasado a veces a referirse genéricamente a 'árbol'). Esta interpretación, que es obvia, se ha rechazado, sin duda, porque en celta, incluido por supuesto el celtibérico, la $p$ - indoeuropea inicial cae. Pero, tras su caída en una parte del celta, desde luego en galo, en britónico y en ejemplos del celta de España, se crearon nuevas $p$-, procedentes de la labiovelar $k^{k}$ (procedente de asimilación). Éste es nuestro caso. Es un cambio fonético an-

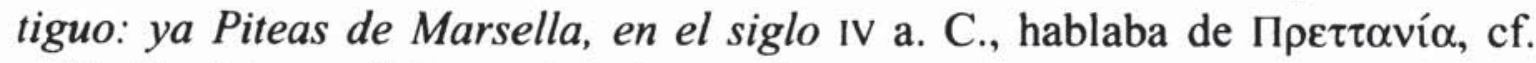
galés Prydein, también pedwar 'cuatro', etc.

Claro que hay el problema, que es sin duda el que ha disuadido de esta interpretación tan obvia, de la presencia en el bronce de la labiovelar, conservada en -cu- (y cue). De ahí que Tovar ${ }^{8}$ hable de la conservación de la labiovelar en el celta marginal (goidélico y celtibérico) frente a la solución $p$ del galo y el britónico. Pero esa conservación es un arcaísmo que se da también en galo, donde hay por ej. sequani, Quanta, Equos ${ }^{9}$. El celta de

situado en algún lugar al pie del Cabezo de las Minas, que corresponde a la acrópolis de la ciudad celtibérica.

En las inscripciones grecoceltas hay un concepto similar, el de $\delta \rho v v \varepsilon \mu \varepsilon \tau o v$, lugar sagrado relacionado con el arbolado: cf. Nemetobriga en León, diversos topónimos en las Galias, por ej., Nemetodurum y palabras del celta insular como air. nemed, agall. nivet 'lugar

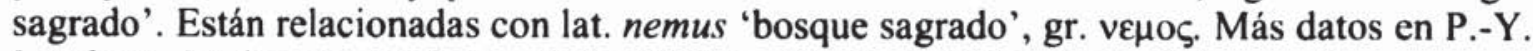
Lambert, La langue gauloise, París 1994, p. 85. Es notable el paralelo con leges sacrae romanas en que se prescriben multas o sacrificios expiatorios para quienes violen de diversas maneras un lucus, cf. J. F. Eska en ZCPh 43, 1983, p. 216 ss.

${ }^{8}$ "Indogermanisch, Keltisch, Keltiberisch», en Indogermanisch und Keltisch, Wiesbaden 1977 , p. 53.

9 Cf. J. Gorrochategui, «Descripción y posición lingüística del celtibérico», en Memoriae L. Mitxelena magistri sacrum, San Sebastián 1991, p. 13 (nótese que ${ }^{*} k^{*}$ evoluciona igual que $\left.{ }^{*} k^{*}\right)$. 
España lo mantiene, pero al lado presenta la innovación $p$, véase a continuación.

Hay que proponer, en efecto, como digo, una asimilación perk ${ }^{\mu}>q^{\nu} e^{k} k^{\mu}$ sin entrar en la uexata quaestio del ítalo-celta, es bien claro que en ambos dominios lingüísticos se da. En ambos hay, por ejemplo, ${ }^{*} k^{u} e n k^{u} e$, de ${ }^{*}$ penk $k^{u} e$ (cf. lat. quinque, galo pinpetos y petru-, en España occidental Pentius, Pintius ${ }^{10}$ ); y en España, en derivados de la palabra que nos ocupa, hay la asimilación en Querquerni, Quarquerni ${ }^{\prime \prime}$. No hay, pues, argumento alguno para negar que en celta, a más de la forma con * $p$ - (caída en 'Аркоv́vı ǒ $\eta$, Hercynia silua), pudiera haber la forma con ${ }^{*} k^{*}$ - asimilada, como en lat. quercus. Y no está excluida, pese a Walde-Pokorny, etc., que el dios lituano Perkúnas, unido a la encina y el rayo como Zeus en Dodona, tenga que ver con esto ${ }^{12}$. Se trataba, en efecto, en Botorrita de un bosque sagrado de encinas u otros árboles. Bosque de los dos dioses ya citados y que estaba sometido a las regulaciones de la lex sacra. Et percunetacam es, ya dije, uná sustantivación: el 'dominio o territorio sagrado del encinar', digamos.

Porque percuneta era, efectivamente, un colectivo (Meid, p. 103), un equivalente en ${ }^{*}-\bar{e} t a \bar{a}$ de los colectivos arbóreos a que se refería el latín -ētum: uiretum, arboretum, pinetum, spinetum, fraxinetum, etc. Nótese que árboles en -nus o -nā forman el colectivo en -netum. Ni más ni menos que como de un nombre de árbol que sería en celtibérico percuna, supongo, salió el colectivo percuneta 'encinar'. Y de él el derivado en $-k \bar{a}$, sustantivado, ya dije, como 'el dominio del encinar' de Togoits y Sarnicio.

Pues no es lo mismo percuneta que percunetaca, como no era lo mismo Lutia que lutiacos, arekorata que arekoratikos: el encinar daba nombre al dominio, denominado por él con una sustantivación. El dominio era a todas luces más amplio, a juzgar por el texto, que habla de sembrados y de construcciones.

Con esto queda aclarado, creo, el inicio de la inscripción: una indicación locativa: T., dominio del encinar de los dos dioses; una indicación de que el senado o cuerpo legislativo decidió; y una prohibición genérica que anticipa las particularizadas, con la fórmula que traduzco por nefas y que encuentra paralelos normales en indoeuropeo ${ }^{13}$.

Creo que ésta es una solución simple, económica y muy propia del estilo de estas inscripciones y regulaciones.

${ }^{10} \mathrm{Cf}$. J. Gorrochategui, «El celtibérico, dialecto arcaico celta», EMERITA 62, 1994, p. 306.

$"$ Cf. J. Gorrochategui, «Descripción y posición lingüistica del celtibérico», cit., p. 14.

12 Véanse paralelos, también de carácter religioso, en otras lenguas en S. Pokorny, ldg. Etym. Wb., Berna 1959, p. 822.

${ }^{13}$ Cf. F. Villar, art. cit., p. 377. 
3. To, [u]ertaunei, taunei, tisaunei y śos aucu areśtalo tamai en la unidad II de la inscripción

Sobre el sentido general de esta unidad, excepto las cuatro últimas palabras, tan oscuras, se está generalmente de acuerdo: el nelitom 'nefas' que concluye la primera unidad se especifica con tres prohibiciones unidas copulativamente con cue: ' $n e+$ infinitivo + fas' es el esquema de cada una de ellas. Hay una serie de comportamientos que no son admisibles en relación con el dominio sagrado cuyo uso es regulado. El problema es el significado de los tres infinitivos. Y el del to inicial que, evidentemente, se refiere a la totalidad (y el maśnai bastante enigmático que precede al tercer infinitivo).

Es claro que los dos primeros infinitivos son del mismo verbo, sólo que el primero lleva un preverbio [u]er procedente de IE *uper, como es bien sabido. Meid prefiere la etimología de la raíz a partir de ${ }^{*} d h \bar{e}$ 'poner' en su grado 0 *dho seguido de un alargamiento -un. To sería de un adverbio de la raíz pronominal bien conocida. Y el tercer infinitivo, tisaunei, vendría de ${ }^{*} d \bar{e}$ (cosa comúnmente admitida) y (siguiendo a Eska ${ }^{14}$ ) un infinitivo ${ }^{*}$ sā-un-ei de la misma raíz del lat. sānus. Para maśnai sigue a K.-H. Schmidt ${ }^{15}$ : sería un D.-Instr. de *mad-snā, de la raíz celta *mad 'romper'. La traducción que propone es:

"Weder es ist erlaubt, dort (?) (etwas) daraufzutun, noch es ist erlaubt (Arbeiten) zu verrichten, noch ist es erlaubt, durch Bruch Schaden zu verüben».

No son, ciertamente, las primeras prohibiciones que esperariamos en un texto como éste. Y hay otras dificultades. El que vaya el mismo verbo dos veces seguidas, la primera con *uper, la segunda como simple, indica una diferencia u oposición buscada, que no se ve en la arbitraria traducción que nuestro autor da en los dos casos a la raíz *dhe. Esta raíz, de otra parte, no la conocemos ampliada con -un. Pero sí sucede así en el caso de *do 'dar' (grado

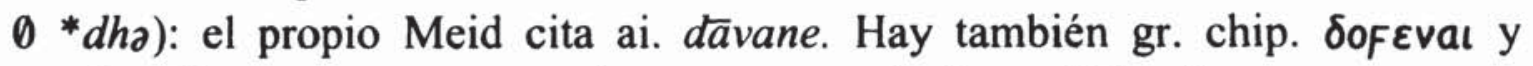
muchas formas en que esta raíz aparece con la forma ${ }^{*} d a{ }_{\alpha}{ }^{16}$.

14 J. F. Eska, Towards an interpretation of the Hispano-Celtic Inscription of Botorrita, Innsbruck 1989.

15 K.-H. Schmidt, «Zur keltiberischen Inschrift von Botorrita», Bulletin of the Board of Celtic Studies, 26, 1976, p. 385.

16 Véanse en mis Estudios sobre las sonantes y laringales indoeuropeas, 2." ed., Madrid 1973, p. 410 (citados en adelante como Laringales). Añádase, quizá, el lepóntico tetu < *dedou, cf. M. Lejeune, "Celtibère et lepontique», en Homenaje a Antonio Tovar, Madrid 1972 , p. 266. Se recordará que, según mi teoria, se trata de formas fonéticas, puesto que reconstruyo la forma antigua de la raiz como ${ }^{*} \mathrm{deH}_{3}$ (véase la última exposición en Laryngale mit Appendix?, Innsbruck 1994). Pero es indiferente a nuestro tema que se acepte esto o que se siga pensando en un alargamiento: el caso es que las formas existen. 
¿Por qué, entonces, negarse a admitir aquí el verbo 'dar', que ya propuse en mi trabajo anterior ${ }^{17}$ y que aparece en todo caso en la inscripción (VI tatu's)? Posiblemente precedido por *uper es 'dar a cambio', como vं $\varepsilon \varepsilon \rho \delta i ́ \delta \omega \mu$ en Eurípides, Fr. 360. 18 (aunque también podría pensarse en un sentido emparentado con el de lat. perdere 'arruinar'). Se prohíbe, pues, cambiar las tierras en cuestión por otras de fuera y se prohíbe también darlas, regalarlas. Deben mantenerse en poder de la comunidad que las administra, el koinón o anfictionía, para expresarme en términos griegos.

$\mathrm{Y}$ creo que esos dos verbos (y el siguiente) tienen un complemento to 'ello', que de un modo vago designa en neutro el total del témenos. Que ese complemento es necesario se ve porque Meid lo tiene que suplir con su «(etwas)». Innecesario suplirlo: está en el to.

Finalmente, defiendo también mi anterior propuesta respecto a tisaunei: viene de ${ }^{*} d \bar{e}$ y del verbo ${ }^{*} s \bar{e}(u)$ 'arar', del cual aparece en otro lugar de la inscripción la forma sisonti, que se compara con lat. serunt. Ese verbo, como el anterior, presenta formas abundantes con $-u^{18}$.

¿En qué consiste la prohibición, pues? Parece que en 'desembrar', esto es, arruinar la siembra, arrasar las tierras. 'Por la fuerza', si se acepta la interpretación de maśnai dada arriba. Que en ellas se siembra se deduce del texto que sigue.

Se prohíbe, pues, en definitiva, permutar, donar o arrasar las tierras del témenos. Es lo primero. Algo muy lógico en un documento como éste ${ }^{19}$.

Y con esto pasamos a la conclusión de la unidad: śos aucu areśtalo tamai.

Meid (pp. 38 y 88) piensa que esta frase debe introducir excepciones o regulaciones más detalladas: acepta la interpretación de aucu como *aukte (cf. gr. $\alpha$ ṽ $\tau$ ) de Eska ${ }^{20}$, aunque con el sentido de 'ausser' y no de 'und, auch', interpretación seguida también por Hamp ${ }^{21}$ ('and furthermore', 'moreover').

Pienso que esto es lo correcto: al final del proemio se introduce una especificación y todo concluye, en IX, con la promulgación del decreto por el magistrado principal. La frase final de II, por tanto, anuncia o abre las series de las reglamentaciones, y debe entenderse como una frase nominal con un (es) elíptico: ni más ni menos que como en I el nelitom final abre la serie de las prohibiciones de II. No hay, pues, la dificultad sintáctica a que alude Meid, ni parece viable su 'salvo con permiso (tamai) de la autoridad (areśtalo)'. Pienso que prohibiciones como las que preceden no toleran excepciones.

17 Cf. Adrados, art. cit., p. 32.

18 Cf. mis Estudios sobre las sonantes y laringales indoeuropeas, Madrid 1973, p. 424 s. (het. sehur, lat. seui, ags. sāwan, anord. saurr, etc.).

19 Para otra interpretación, cf. E. P. Hamp, «Botorrita [u]ér taunei... taunei... tisaunei», Veleia 7, 1990, pp. 165-166.

20 J. F. Eska, Towards an interpretation..., cit., p. $51 \mathrm{~s}$.

21 E. P. Hamp, «Botorrita śos aucu / are[i]talo tamai», EC 27, 1990, pp. 179-180. 
Creo, pues, que el aucu que sigue al demostrativo śos es un adverbio o partícula y no un sustantivo, como proponen Eichner y Villar. 'Este dominio', traduce Eichner 22: 'dieser Hain (gehört) der *dāmā des Erstgenannten'); 'this decree shall be put in the place', traduce Villar ${ }^{23}$. Pero no veo argumentos para hacer de aucu un nombre con uno de esos significados, ni para hacer de la palabra que sigue (que tiene, ciertamente, dificultades de lectura) un verbo ${ }^{24}$.

Pero es buena la intuición de Villar de que en este momento una alusión al propio decreto es esperable: es algo frecuente no sólo en inscripciones griegas y latinas (él da ejemplos de éstas, con hoce seignum, hoc monumentum), sino también en las indias de Aśoka. Si aucu es un adverbio que introduce un nuevo tema, el sustantivo que va con śos debe de ser arestalo, cuya etimología está bien establecida, pero no el sentido: viene de la bien conocida preposición celta

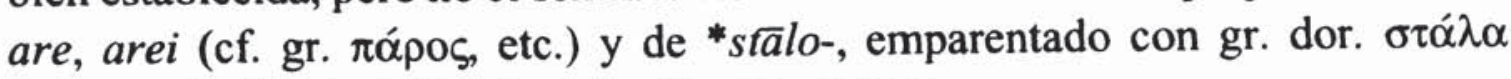
'columna'. ai. sthāla 'puchero', gót. stols 'silla'.

Es cualquier cosa que está delante de la estelą o cipo o muro: la plancha de bronce o inscripción sin duda, el «decreto» traslaticiamente. Efectivamente, el bronce, que se halló en una casa particular sin duda como resultado de un traslado, debió de estar sujeto en algún momento a un elemento arquitectónico fijo ${ }^{25}$ : de ahí su nombre de areśtalo 'lo que está delante de la estela o muro'.

Por tanto, siendo tamai un Dat. de la primera declinación, la traducción no puede ser sino: 'y esta (es) la regulación para...' (salvo que se trate de un instr., cosa menos probable). Evidentemente, tamai debe referirse a la comunidad cuyo santuario está en Tricanta: debe ser, pues, como Eichner por ejemplo

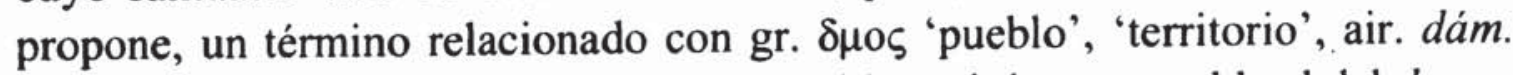
'grupo', 'pueblo' (al. «Gefolgschaft»). Sentido, quizá, comparable al del da-mo de Pilos, mencionado igualmente en una regulación del uso de tierras de un santuario que le eran cedidas ${ }^{26}$.

Traduzco, pues:

Hoc (est) autem decretum populo

${ }^{22}$ H. Eichner, «Damals und heute: Probleme der Erschliessung des Altkeltischen zu Zeussens Zeit und in der Gegenwart», en Erlanger Gedenkfeier für Johann Kaspar Zeuss (ed. B. Forssman), Erlangen 1989, p. 45 ss.

${ }^{23}$ F. Villar, "Botorrita soz auku aresta[.][.] tamai», en Sprachen und Schriften des antiken Mittelmeerraumes. Festschrift für J. Untermann zum 65. Geburtstag (eds. F. Heidermanns, H. Rix y E. Seebold), Innsbruck 1993, pp. 465-471.

${ }^{24}$ Villar compara subjuntivos perifrásticos con -el en celta insular. No hay base para atribuir esto al celtibérico.

${ }^{25}$ Cf. Beltrán-Tovar, Contrebia Belaisca, cit., pp. 33 y 35 .

26 Véase mi último tratamiento del tema en «Notas de entrega, no catastro en Pakijana», en prensa en las Actas del II Congreso Internacional de Micenología (Roma 1991). 


\section{Observaciones a la unidad III de la inscripción}

Pienso que, en líneas generales, se ha llegado a una interpretación correcta, aunque Meid vacila entre entender oścues śtena uersoniti como 'el que viola esas (prohibiciones)' o 'el que realiza esas actividades': después de lo dicho, se impone la segunda interpretación, que corresponde bien al sentido de la raíz (cf. ai. sanóti, het. śanh-, gr. $\dot{\alpha} v v ́ \omega$ ). Pienso acertada la interpretación de lo que sigue por Meid: el que realiza esas acciones debe tomar (capiseti) una determinada cantidad de plata acuñada para entregarla (otanaum) en el templo de Tocoits (tocoitei eni).

Mis observaciones son dos:

1. Señalo la correlación uta (inicial de III)... uta (inicial de IV)..., seguidos los dos de óscues. Viene a equivaler a correlaciones bien conocidas en otras lenguas y debe traducirse por 'tanto el que... como el que...', seguido de verbos personales que indican la acción que se atribuye al respectivo sujeto (uersoniti y ampitiseti). La diferencia es que en III la oración principal lleva un verbo en subj. (capiseti... otanaum 'debe tomar... para dar...'), mientras que en IV lleva un imperativo (uśapitus).

2. Esto me lleva a hacer algunas indicaciones sobre los modos y tiempos verbales. En la oración subordinada que abre IV y también en la de $\mathrm{V}$ hay claramente un subj. con $*_{-}-\bar{a}$ o con $*_{-s}$ (III oścues ampitiseti, V iom aśecati, VI iaś... ropiséti). ¿Qué hacen, entonces, indicativos en oraciones paralelas como la de III oścues... uersoniti y VI iomui... sisonti, iom... pionti, quizá VIII ioś... auseti? ¿O no son indicativos? ¿Y qué valor exacto tiene el subj. capiseti de III? Creo que es éste un tema importante para la morfología indoeuropea.

Meid, p. 110, ofrece una discusión circunstanciada de sisonti, que él cree que no es como se dice generalmente un ind. equivalente al lat. serunt, sino un subj. con vocal temática de un ind. atemático $\left({ }^{*}\right.$ sisēmi). Pero yo ofrezco una solución más simple: todas estas formas personales con $*$ elo son arcaísmos que equivalente tanto a ind. como a subj.: estaban todavía indiferenciadas modalmente ${ }^{27}$.

Para la teoría general de cómo el subj. se diferenció del ind. oponiendo, de entre los temas primitivamente sin definición modal, uno a otro, remito a mis obras anteriores ${ }^{28}$. Es una cuestión de oposición: las formas temáticas se hacen subj. frente a un ind. atemático, ind. frente a otros temas hechos de subj. (lat.

27 Igual es el caso, pienso, de III uersoniti y de VI cuati. Sobre la flexión verbal en celtibérico preparo un trabajo independiente, que aparecerá en un libro de Homenaje al profesor Kuryfowicz.

${ }^{28}$ Cf. Evolución y Estructura del Verbo Indoeuropeo, 2." ed., Madrid, CSIC, 1974, p. 849 ss.; Lingüistica Indoeuropea, Madrid, Gredos, 1975, p. 719 ss. 
legis/legās, pero cantās/cantēs y monēs/moneās). Pero en fecha antigua no había oposición ind./subj.: no la había en hetita, báltico y eslavo, no la había en ciertos arcaísmos aislados en las distintas lenguas. Por ejemplo, en air. había formas con $-{ }^{*} \bar{a}$ de ind.-subj. ${ }^{29} \mathrm{e}$ igual en osco-umbro y en germ. (con ${ }^{*} \bar{a}$ y

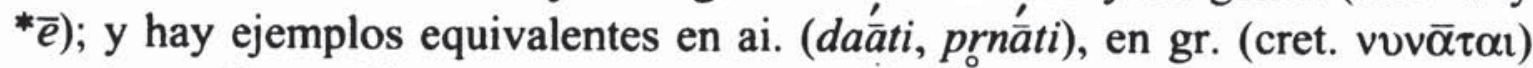
y, sobre todo, en diversos temas del tocario ${ }^{30}$. En esl. y bált. falta en absoluto un subj. diferenciado.

Pero, sobre todo, en indio antiguo quedan huellas muy claras de formas temáticas indiferentes a la oposición ind./subj. ${ }^{31}$ Son absolutamente idénticas a las que hallamos en celtibérico y que, en las subordinadas relativas o condicionales a que he hecho referencia, funcionan como subjuntivos. He aquí, pues, un arcaísmo más de los conservados en las zonas marginales del IE, el indio y el celta peninsular en este caso.

En cuanto al capiseti de III silapur capiseti, puede entenderse como subj. que coja plata. Pero se esperaría más bien un impvo., como en la frase paralela (IV úsapitus). Es claro que ese subj. está próximo en este pasaje a un fut., como en tantos futuros en $-s$ - (lat. erit. < *eseti, gr. ह̌ $\sigma \varepsilon \tau \alpha l$, etc.) ${ }^{32}$. IV pisetus parece una forma de impvo. formada sobre un futuro de este tipo; hay otra comparable de un subj. con $-a$, a saber, $\mathrm{V}$ oiśatus.

\section{Observaciones a la unidad IV de la inscripción}

Comienza con uta oścues... ampitiseti: 'y cualquiera que' + complementos directos (véase más abajo) + ampitiśeti. El verbo, de la raíz * dheigh 'trabajar

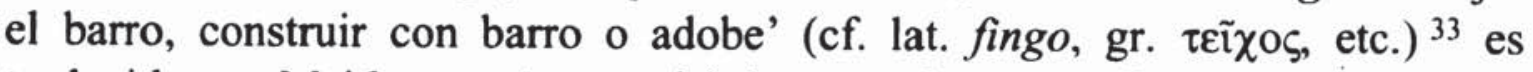
traducido por Meid como 'construir': los complementos directos son boustom 'un establo' (interpretación bien establecida) y otros menos claros.

Aquí tengo que hacer dos observaciones:

a) El subj. ampitiśeti no puede ser simplemente «errichten», 'construir': aquí y en $\mathrm{V}$ ampitincounei (inf. del mismo verbo en pres.) el preverbio ampi tiene claramente un valor "en torno' ${ }^{34}$. Cf. av. pairi-daēza- 'muro en torno', transcrito en gr. como $\pi \alpha \rho \alpha ́ \delta \varepsilon ı \sigma o \zeta$. Se trata de alguien que cerca con un muro

29 Cf. R. Thurneysen, Handbuch des Altirischen, Heidelberg, Winter, 1909, p. 356.

${ }^{30}$ Cf. mi Verbo Indoeuropeo, cit., p. 849 ss.

${ }^{31}$ Cf. Verbo Indoeuropeo, cit., p. 280 (doctrina procedente de Renou).

${ }^{32}$ Sobre el futuro sigmático del air., cf. Thurneysen, ob. cit., p. 379 ss. La discusión sobre si viene de un subj. o de un desiderativo, es bastante estéril.

33 Imposible, pues, establecer dos raíces distintas, como propone K.-H. Schmidt, «Zur Keltiberischen Inschrift von Botorrita», The Bulletin of the Board of Celtic Studies 26, 1976, p. 382.

34 Y no 'de nuevo' ('rebuild' según Eska, ob. cit., p. 43). 
de barro un establo y otras instalaciones más y que, por ello, es obligado a 'excavar', construir un camino (camanom uśapitus, en impvo.).

b) En cuanto a los complementos directos que siguen a boustom, dejo coruinomue, sobre el que no puedo aportar nada. En cuanto a macasi [a? ?mue, que sigue, recuerdo mi propuesta ${ }^{35}$ de que es un derivado de maga 'campos', en relación con magus 'campo' de la inscripción lusitana de Lamas de Moledo. Y para el final ailamue vuelvo a la vieja interpretación de Tovar ${ }^{36}$ de que se trata de un equivalente de lat. aliamue 'y otra'.

Los hechos léxicos y fonéticos son bien claros: cf. galo Allo-broges, air. Ac. pl. firu aile 'otros varones', galés ail, etc. La objeción de K.-H. Schmidt, aceptada tácitamente por Meid ${ }^{37}$, es de orden sintáctico: se espera que los ue hagan concordar sustantivos y ailam 'otra' es un adjetivo. Pero no creo que sea decisiva.

Pienso, efectivamente, que hay una correlación de tres tipos de instalaciones unidas con ue, y que de ellas la tercera, macasi $i[a] m$, sea buena mi etimología o no, es un nombre genérico determinado por ailam 'otra': "un establo o un... $\mathrm{u}$ otra instalación agraria». Sobra, simplemente, el último $u e$, que ha sido añadido mecánicamente por el grabador.

En fin, éste es un detalle menor. El hecho es que cualquiera que obstaculice los accesos ( ¿al templo?) al cercar diversas instalaciones, debe 'excavar' un camino: verbo uśapitus (un impvo. de $p i<{ }^{*} b h \bar{\imath}$ ), con un preverbio *eks o*ups) que se opone al tinpitus de VI (de *dê-en), de que hablaré más abajo.

Pero la unidad se cierra con una frase bastante misteriosa, osaś śues śailo cuśta pisetus. Suele entenderse que osas śsueś es el complemento directo, que el sujeto es cuśta y que de él depende un Gen. śailo. Hasta aquí llega el acuerdo. Pero ninguna de estas palabras es clara.

El verbo es, evidentemente, pisetus que al ser transitivo debe de ser 'golpear', con el sentido concreto que sea, no 'ser': son las dos interpretaciones vigentes, que parten respectivamente de *bhei y *bheu, la primera está apoyada por repetidas apariciones de $p i$ en nuestra inscripción. El sujeto es custa, sin duda un abstracto femenino en ${ }^{*}-\bar{a}$ relacionado con VI cuśtaicoś... cuati, que parece ser 'un inspector... inspecciona', todo ello a su vez en relación con lat. custos, etc. Creo que el cuśta de nuestro pasaje, sujeto de una acción como

${ }^{35}$ Cf. Adrados, art. cit., p. 37.

36 A. Tovar, "Las inscripciones de Botorrita y de Peñalba de Villastar y los límites orientales de los celtíberos", Hispania Antiqua 3, 1973, p. 381 s. También otros autores, cf. Eska, ob. cit., p. 38.

${ }^{37}$ K.-H. Schmidt, art. cit., p. 381; Meid, pp. 48 y 80 (él mismo reconoce que *auila 'establo de ovejas' (lat. ouile) no es fonéticamente posible. 
'golpear' o quizá 'sacrificar', más que 'necesidad' o una cosa así como propone Meid, es 'la guardia', referido a un cuerpo de guardia o a un individuo ${ }^{38}$.

La existencia de un custaicoś que es un funcionario hace ver bien claro que cuśta es un N. sg. fem., no un n. pl. como se ha propuesto a veces. Hay paralelos numerosos en celta de España en que un nombre en - $a$ crea un derivado -aico ${ }^{39}$. En suma: hay un colectivo o un individuo que toma de él su denominación y que ejerce alguna acción sobre quien ha hecho algo delictivo, aparte de la obligación que ya se le ha impuesto. Esto está en relación con VI cuśtaicoś... cuati, un funcionario ejerce a su vez una función de inspección. El dominio sagrado tenía a su servicio funcionarios que velaban para que la reglamentación se cumpliera.

Esto para mí es muy claro. Mucho menos lo son los otros dos puntos:

a) El complemento directo osaś śueś. La interpretación de śueśs como posesivo nos deja sin un nombre para ese adjetivo: el propio Meid así lo reconoce, llega a decir (pp. 51 y 116) que funciona como un nombre femenino y traducir 'alle solche (Arbeiten)' no le satisface.

Mis propuestas son, en este caso, puramente hipotéticas. Pienso si śueś tiene que ver con *s $\bar{u}$ - 'cerdo' (cf. galés $h w$, air. socc-) y el verbo pisetus, extraña forma que es un impvo. derivado de un futuro (véase más arriba) es 'matar', 'sacrificar': aquel que había hecho aquellas cercas ilegales no sólo tiene que abrir un camino, también hacer un sacrificio de 'esos cerdos', un número sin duda conocido. Por lo demás, no es claro el Ac. en -eś (tampoco si se parte del posesivo, como se suele). En fin, podría volverse a la interpretación de sueś como 'seis' (*sueks), pero osaś sigue siendo problema.

b) El Gen. determinante śailo. ¿De quién es guardián el cuśta? Aquí otra hipótesis, que sólo a título de tal presento. Meid, p. 111, propone śailo como Gen. de *sa(u)ilo, que sería «Werk, Vollendung». A mí esa forma me recuerda la de la palabra del «sol»: un *saulio-> *sauilo-> *śailo- parece fonéticamente correcto. Compárese galés haul, acorn. heuul, por no hablar de formas como gr. $a_{F} \varepsilon \operatorname{lio\zeta }^{40}$. Nos hallaríamos, pues, ante una guardia o un guardia o custodio del dios Sol. Quizá sacrificaría a éste los cerdos ofrecidos por aquellos que construyeran aquellas cercas ilegales.

${ }^{38} \mathrm{La}$ interpretación viene de L. Fleuriot, "La grande inscripción celtibère de Botorrita», $E C$ 14, 1975, p. 428. Da paralelos celtas. La crítica de Eska me parece poco convincente: rompe conexiones evidentes.

${ }^{39} \mathrm{Cf}$. U. Schmoll, Die Sprache der vorkeltischen Indogermanen Hispaniens und das Keltiberische, Wiesbaden 1959, p. 54 ss.

${ }^{40}$ En mi teoría laringal (cf. Laringales, cit., p. 425) la raiz es * ${ }^{*} H^{*} 3$, de donde saldrían en grado pleno tanto las formas con $\bar{o}$ (lat. $s \bar{o} l$, aisl. $s \bar{o} l$ ) como las con $\bar{a} u, \bar{a} u$ (las citadas y otras como gót. sauil). 
Interpretación, insisto, hipotética, que propongo porque la de Meid 'das, was Notwendigkeit des Werkes ist, darf er (um-, weg-)hauen' no me satisface.

\section{Observaciones a las unidades $V$ y VI de la inscripción}

Para $\mathrm{V}$, parece aceptable sustancialmente por ahora la interpretación de Meid, que implica una condicional: 'Wenn er (Erde) aussticht zum Zwecke des Bauens, dann soll er diese (Materidlien) innerhalb von 3 Tagen (?) aus der Umhegung herausschaffen', corrigiendo naturalmente la interpretación del ampitincounei: se trata escombros que son resultado de hacer aquellas cercas ilegales. Puede haber, ciertamente, algunas dudas sobre el iom. Y tiene interés señalar la oposición de los proverbios (uśapitus/tinpitus) y de los temas verbales (ampitiśetilampitin-counei).

Donde creo poder añadir algo es en la interpretación de la frase final neito tiricantam eni oiśatus, en que los intérpretes ven un dios Neitos, cuyo papel aquí (¡recibir escombros!) no veo claro. En mi trabajo anterior propuse que neito es un impvo. 'que no vaya', interpretación que Villar ha recogido últimamente ${ }^{41}$. Se le dice a ese infractor 'que no vaya a Tiricanta, que se los lleve (los escombros lejos)', lo último siguiendo la interpretación más aceptada.

También creo que estamos acercándonos a una interpretación satisfactoria de VI: hay gentes que tienen que pagar diezmos a aquel para quien siembran (sisonti) ciertos campos, pero sólo si (iom) si recolectan las cosechas y si un inspector inspecciona las que siegan 'fuera' o 'dentro'. Es notable la oscilación entre el verbo en sg. y pl.: hay implícito un sujeto plural, pero también uno indefinido, como el oścues de más arriba.

Ciertamente, quedan algunas dudas y entre ellas, sobre todos, liśtaś titaś, el complemento directo de sisonti: los campos cultivados por los que se pagan diezmos tras cumplir ciertas condiciones ${ }^{42}$. No quiero entrar en la discusión sobre lo que significa exactamente la expresión: en todo caso, tiene un sentido jurídico, se refiere a un tipo especial de tierras dentro del dominio, tierras que son cedidas en ciertas condiciones ('tierras asignadas', de *dhə?). Es, pues, un tercer punto de la reglamentación, que sigue al primero (prohibición de dar,

${ }^{41}$ Cf. Adrados, art. cit., p. 40, F. Villar, "Tratamiento de $-\bar{o}$ en sílaba final. Algunas posibles formas de imperativo en celtibérico", Veleia 6, 1989, p. $202 \mathrm{~s}$. La distinción del impvo. en -to y los en -tus la explica Villar por la caida en estos últimos de una - $o$, cf. "The verbal desinence -tus in the Hispano-celtic inscription of Botorrita", ZCPH 43, 1989, pp. 214-22.

42 Estos «diezmos» (y los mencionados en IX) tienen sin duda una relación con el $\delta \varepsilon \kappa \propto \nu \tau \varepsilon \mu$ de inscripciones galas en que se paga en reconocimiento a ciertas deidades. Cf. Meid, ob. cit., p. 119 y ejemplos de dichas inscripciones en P.-Y. Lambert, La langue gauloise, París 1989, p. 86 ss. 
arrasar, etc., el dominio y castigo correspondiente) y al segundo (reparación que se exige al que obstaculice el paso dentro del dominio).

Ahora resulta que, además, se habla de ciertas tierras cuya explotación se cede, a cambio de un diezmo a pagar a aquél que las ceda. Esto es muy misterioso, pero nos recuerda, grosso modo, la reglamentación de dominios micénicos como el de Pakijana y la de otros orientales que con ellos se han comparado ${ }^{43}$. Hallamos, una vez más, la misma mezcla entre lo sagrado del dominio y su gerencia por un representante del estado: en este caso, el cuerpo de los compalces o bien su jefe (compalcores). Existen, incluso, funcionarios para la administración o la inspección.

Es bien claro que aquellos para quienes 'se siembran' las tierras han de ser por fuerza funcionarios civiles o religiosos o las dos cosas a la vez. No encontramos, como en esos otros casos, una distinción entre tierras del rey o del santuario, de un lado, y otras del 'pueblo', ni una entrega de tierras a cambio de servicios. ¿O es que todo tiene aquí que ver con el 'pueblo' (tamai en II)?

También puede suceder que la distinción entre aquellos que ceden tierras y reciben un diezmo y aquellos que las reciben, cultivan y dan el diezmo, tenga relación con la distinción, en el sistema micénico, entre los que reciben tierras (del palacio o el santuario, pienso) y aquellos a los que dan onata o participación en las mismas. Todo apunta a la existencia de un sistema más o menos semejante.

\section{Observaciones a las tres últimas unidades (VII a X) de la inscripción}

No son muchas mis aportaciones a este sector. Para VII hemos de quedarnos, provisionalmente, con la interpretación a que, tras Hoz y otros, ha llegado Meid, aunque tengamos dudas sobre muchos puntos, por ejemplo, sobre qué significan exactamente ancioś y eśancioś.

No deja esta sección de ofrecer cosas de interés: comienza una reglamentación digamos que "parcial», referida a sectores del dominio (śomei initousei, śarniciei) y a algunos de los pueblos incluidos en el koinón de que depende el santuario: concretamente, los Acaina, que aparecen en una construcción pasiva, acainacupo nepintor. Posiblemente, las regiones calificadas de anciós y ésanciós no deben ser recolectadas (pl.) por los de Acaina.

VIII continúa la "regulación parcial», ahora referida a las inmediaciones o al dominio restringido del otro dios, Tocoits. Aquí es claro que el que realice ciertas acciones tiene que dar un diezmo (tecametam). Esas acciones se refie-

${ }^{43}$ Entre los hetitas, en Ras Shamra y Mesopotamia, también en Chipre. Sobre el tema, véase mi última exposición en "Notas de entrega, no catastro en Pakijana», cit. 
ren o a la tierra arada (aratim) o a otra calificada de urantiom, quizá tierra de pastos.

Aquí el problema es el verbo auseti. Para Meid, pp. 66 y 88, es 'möchte nützen', se trata de aquellos que exploten en su interés ciertas parcelas; compara ai. avati, lat. auere, etc.

Esto es dudoso y hay que reconocer que existe una gran incertidumbre sobre el sentido de este verbo, que es clave para entender el pasaje. Las principales propuestas están recogidas por Eska ${ }^{44}$ : van de 'ór' (Tovar), a 'gedeihen lassen' (Schmidt), 'aceptar' (Fleuriot).

En casos como éste hay que acudir al contexto general. Salvo VI, que habla de permisos de cultivo en ciertos casos y condiciones, el resto de la inscripción se refiere a prohibiciones y castigos por las acciones referidas. Concretamente, en la unidad previa (VII) se hace una de esas prohibiciones, dentro de las «regulaciones restringidas» de que hablamos.

Pues bien, creo que también en VIII hay una prohibición, referida ésta al territorio de Tokoits. El ioś + subj. continúa la serie de las oraciones subordinadas que indican que 'el que, todo el que' haga tal cosa debe dar tal compensación o recibir tal castigo. En cambio VI, que recoge el derecho de algunos a sembrar y recolectar, carece de sujetos: eran sin duda bien conocidos, no había la indefinición de los sujetos ('cualquiera que...') de las cláusulas penales.

En mi trabajo anterior ${ }^{45}$ sugeri 'quemar', aludiendo a gr. દü $\omega$, ai. oṣati, lat. uro. Tenía dificultad fonética. Ahora me inclino al mismo sentido, pero parto de un IE *aus-, que he estudiado detenidamente en otro lugar ${ }^{46}$.

«Quemar» es, pienso, el sentido original de gr. $\alpha v ้ \omega$, relacionado con $\varepsilon v ँ \omega:$ éste viene de un grado pleno $+{ }^{*}$ Heus-, $\alpha$ v้ $\omega$ de uno $\emptyset^{\circ} H u s-$, con vocal protética, igual que en muchos casos paralelos ${ }^{47}$.

En definitiva: el que prenda fuego a estas parcelas próximas a Tokoits, debe pagar un "diezmo» que no podemos precisar.

$\mathrm{Y}$ renuncio a entrar en detalle en la unidad $\mathrm{X}$, que es claramente la proclamación o promulgación del decreto. Quedan en ella cosas dudosas, pero estamos otra vez ante un Consejo que administra los dominios de los dos dioses:

\footnotetext{
44 Ob. cit., p. $52 \mathrm{~s}$.

45 Adrados, art. cit., p. 44.

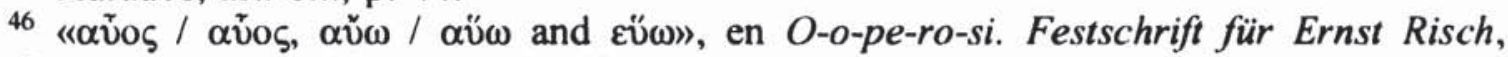
Berlín-Nueva York 1986, pp. 458-462.

${ }^{47}$ Naturalmente, a la misma raíz pertenecen $\alpha$ ṽos / $\alpha$ õos. El ocasional espíritu áspero

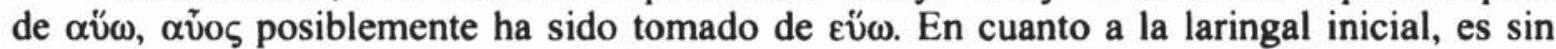
duda $H_{1}$; el apéndice es irreconstituible, según mi teoría se perdía en posición inicial ante vocal. En cuanto a las formas de 'seco' con ${ }^{*} s$ - (lit. saûsas, aesl. suchŭ, i. sósa-, etc.), deben venir de *souso-: el *sausos que se reconstruye es sólo a partir de gr. $\alpha \tilde{o}$, con ayuda de un círculo vicioso. He propuesto *sousos y una raíz independiente.
} 
según la opinión más común, es el jefe (reś) del Consejo el que hace la proclamación; y debe identificarse con apulu upocum y los miembros del Consejo con los que da la cara B. De ahí el pl. del verbo rusimus, que indica la proclamación ${ }^{48}$.

Con esto concluyo. No he intentado más que perfeccionar una interpretación que cada vez va haciéndose más precisa, mediante algunas propuestas que considero verosímiles y algunas sugerencias que quedan meramente como tales. Pero he querido presentar unas y otras recorriendo todo el documento, en un intento de añadir a esas interpretaciones particulares una visión general del mismo.

Es notable que esta visión nos lleve a aproximar su contenido al de documentos similares de fecha mucho más antigua del Mediterráneo oriental: regulación legal del dominio o témenos de un santuario común a varios pueblos (a los de Acaina hay que añadir los gentilicios de la cara B, seguramente), con prohibiciones y autorizaciones precisas vigiladas por ciertos funcionarios, en un contexto entre religioso y civil. Pero también son comparables las inscripciones latinas recogidas por Eska y a las que ya he hecho alusión, en las que se prescriben multas o sacrificios expiatorios para aquellos (si quis...) que violen lugares sagrados denominados comúnmente lucus.

FRANCISCO R. ADRADOS

48 Las dudas que quedan se refieren, sobre todo, a las formas con -rés, cf. F. Villar en su reseña al libro de Eska en $Z C P h 45,1992$, p. 303 s.; y, por supuesto, al carácter de fórmula griega, propuesta por Meid, de aleites iste ireś. En cuanto a rusimus 'proclamamos', a veces entendido como un lat. iusimus, parece estar claramente asegurado como palabra celtibérica de etimología por lo demás no segura (cf. F. Villar, «Las silbantes en celtibérico",, en Lengua y cultura en la Hispania prerromana, Actas del V coloquio..., Salamanca 1994, pp. 773-811). También discuten algunos, como se sabe, la relación entre las dos caras de la inscripción. 